Sebastiano Tafaro

\title{
MEDIAZIONE E PROCESSO: CRISI DEL PROCESSO E RICORSO ALLA CONCILIAZIONE
}

\section{La mediazione nell'UE}

Il nostro tempo assiste alla riscoperta e al rilancio a tutti i livelli sia riguardo al 'pubblico' si riguardo al 'privato' della mediazione.

In particolare si pensa di risolvere le difficoltà di accesso alla giustizia attraverso l'introduzione della mediazione e conciliazione, come forma parallela e stragiudiziale di composizione delle liti.

È questa la soluzione escogitata in sede europea all'interno dell'UE.

L'Unione, infatti, dopo un lungo e faticoso percorso ha emanato una direttiva che si muove in questa direzione e impegna gli Stati membri a introdurre forme di conciliazione spontanea e preventiva delle liti.

Si tratta della direttiva 2008/52/CE del Parlamento europeo e del Consiglio del 21 maggio 2008, entrata in vigore il 12.6.2008, la quale promuove il ricorso alla mediazione come metodo di risoluzione consensuale delle controversie in materia civile e commerciale ed impegna gli Stati membri a realizzarne il contenuto entro maggio $2011 .^{1}$

Poiché le procedure sono diverse da Paese a Paese, pur all'interno dell'Unione, vi sarà un varietà di modalità di applicazione della direttiva, dalle quali dipenderà il raggiungimento effettivo dello scopo perseguito con essa.

Basti considerare un punto. Secondo la direttiva la conciliazione non è obbligatoria, tuttavia se in qualche Stato venga imposta obbligatoriamente prevarrà l'obbligatorietà: ebbene già questo costituisce motivo di differenze fondamentali sull'andamento dei processi.

La direttiva, comunque, rappresenta un esempio del riconoscimento del diritto alla giustizia come diritto fondamentale e segna un passo avanti rispetto alla Carta 
dei diritti fondamentali dell'UE del 2000, nella quale il diritto alla giustizia, previsto al capo VI, era considerato nell'ottica dell'organizzazione tradizionale dell a giustizia, vale a dire quella dei Tribunali, con attenzione accentuata sulle procedure penali. ${ }^{2}$ La nuova direttiva si fonda sulla raggiunta consapevolezza che le difficoltà di accesso alla giustizia in campo civile e commerciale ledono diritti fondamentali e possono essere non meno devastanti di una condanna penale.

L'Ue, con questa direttiva, si fa carico dell'esigenza di assicurare un giudizio di equità a parti che possono avere peso e capacità di organizzarsi nel processo molto differenti. ${ }^{3}$

Il giudizio di equità proposto attraverso la mediazione mi sembra dettato da diverse consapevolezze, dipendenti dai cambiamenti dei sistemi giuridici nel momento attuale. Intanto perché vi è un'obiettiva difficoltà a individuare le norme da applicare. Poi perché, specie nelle materie contrattuali, assistiamo a un'ampia formazione di diritto spontaneo, di difficile conoscenza e dettato da gruppi di potere (e di pressione) molto forti e sovranazionali. Infine perché non si può essere certi della 'giustezza' delle leggi, peraltro spesso di difficile individuazione perché si devono applicare ad una negoziazione che travalica i confini degli Stati, rendendo arduo dire se utilizzare la legge dello Stato A o dello Stato B, di fronte alla globalizzazione dei processi di produzione e di commercializzazione delle merci, i quali interessano piú Stati.

Se questo, come a me pare, è il punto di partenza per ritenere meglio confacente la mediazione (delegata a persona che, presumibilmente, è ritenuta affidabile e riscuota la fiducia di tutte le parti), a me sembra anche che essa non possa risolversi soltanto in un problema settoriale, concernente il processo e/o i mezzi extragiudiziari di risoluzione delle controversie. Essa diventa espressione di una visione che investe il modo di concepire l'organizzazione della società (cioè dello Stato e delle aggregazioni di Stati) a tutto tondo, ivi compresi gli aspetti legati all'amministrazione della Giustizia.

In altre parole, le motivazioni che oggi giustificano il ricorso alla mediazione mettono in discussione il modello di organizzazione dello Stato, non foss'altro che per il fatto che i modi d'interazione dei cittadini con le istituzioni, impostati al modello della democrazia rappresentativa, sono in crisi, sicché di ciò risente anche la Giustizia (tout court) e l'accesso alla Giustizia.

\section{Precedenti}

La direttiva europea 2008/52/CE è eco di questo malessere, ma solo parziale. Essa risente dell'impronta che la mediazione aveva ricevuto nelle esperienze anglo- 
sassoni, dove era nata con riguardo alla soluzione delle dispute destinate al processo, ${ }^{4}$ e sembra orientata esclusivamente alla proposizione di soluzioni da affiancare, sia pure in via alternativa, ai Tribunali. Va però aggiunto che essa, in qualche modo, accoglie l'eredità francese, dove con la rivoluzione francese furono introdotte forme di conciliazione obbligatoria e preventiva delle controversie. ${ }^{5}$

Il richiamo delle difficoltà riscontrati nell'applicazione della mediazione in Francia, dove la conciliazione ha avuto origini proprie, evidenzia la sostanziale e radicale differenza che esiste tra le esperienze di Common Law e quelle di Civil Law: a differenza di quanto si verifica nell'esperienza dei paesi anglosassoni, nei paesi di civil Law vi è una diffidenza (a volte celata) verso la conciliazione. Perciò la direttiva europea mi sembra piú un atto di ottimismo che non uno strumento per la soluzione effettiva e quantitativamente significativa delle controversie.

Per una riflessione consapevole e completa credo che gioverebbe anche richiamare la secolare esperienza del diritto romano.

Il diritto romano, durante la sua lunga storia, ha considerato il processo civile come regolamentazione privata degli assetti di interessi contrastanti: private erano le parti, privato era anche l'organo giudicante (arbiter o iudex). La Repubblica si preoccupava, attraverso un proprio magistrato (principalmente il praetor) di garantire il corretto svolgimento della procedura e l'imparzialità del giudicante.

Nelle diverse fasi che caratterizzarono il processo dell'esperienza romana ebbe sempre grande rilievo la mediazione ${ }^{6}$ : infatti, "L'uso dei privati di affidare la decisione delle controversie ad arbitri, al di fuori del processo o dei processi controllati dagli organi della collettività, fu certamente a Roma molto antico... bisogna credere ... che il pretore abbia modellato il processo formulare che andava sorgendo per suo impulso su un tipo di arbitrato"? Il ricorso all'arbitrato si presentava come una forma di autogestione dei propri interessi e, in ultima analisi, può essere considerato come una faccia della sovranità diffusa riconosciuta ai cives, poiché “... è possibile pensare che la diffusione dell'arbitrato, più che al favore o disfavore del legislatore $\mathrm{e}$ dei minori o maggiori vantaggi rispetto al processo ufficiale, sia dipesa a Roma dall'esistenza di formazioni sociali libere e spontanee, in cui i membri si identificavano più che rispetto alla compagine statale, ed erano tra loro spontaneamente solidali. Si tratta dello stesso fenomeno, in fondo, per cui per lungo tempo il cittadino romano nel compiere un affare ritenne di essere largamente garantito dalla fides risposta nell'altro contraente". ${ }^{8}$ Una delle forme di maggiore diffusione della mediazione consistette nel compromissum, il quale ricorda il moderna 'arbitrato', essendo strutturato sulla promessa di demandare ad un arbitro liberamente scelto la decisione della controversia, a pena del pagamento di una somma di denaro (poena) da parte di chi si fosse sottratto a tale procedimento. ${ }^{9}$ 


\section{La disciplina italiana}

Né di questi precedenti né delle finalità perseguite in sede europea mi pare tener conto la recente introduzione della mediazione-conciliazione nel sistema di composizione delle liti in Italia. A dir il vero, mi pare, che la soluzione italiana, in relazione, alla direttiva europea approdi ad un vero 'pasticcio', per giunta minato da prospettive di facili guadagni a favore di sedicenti organismi nascenti in vista dell'attuazione della mediazione.

La mia pessimistica previsione parte dal parziale fallimento della 'mediazione' già esistente in Italia prima della direttiva: esso investe i pregressi conciliatori ed i giudici di pace, oggetto di continue riforme che, piano piano, li configurano sempre piú giudici giusdicenti per questioni ritenute di lieve entità (in realtà non sempre ciò è esatto!).

Per un certo tempo si è ritenuto che la stessa Costituzione sia d'ostacolo all'imposizione di tentativi di conciliazione obbligatori, sulla scorta dell'art. 24: Tutti possono agire in giudizio per la tutela dei propri diritti ed interessi legittimi. La Corte Costituzionale, però, ha superato questo preliminare impedimento, sostenendo l'obbligatorietà e la piena legittimità costituzionale della conciliazione in materie private (Corte Cost. 13 luglio 2000, n. 276, in FI 2000, I, 2752), sulla base della considerazione che, se è vero che la Costituzione afferma solennemente l'effettività della tutela giurisdizionale, è altrettanto vero che "l' ineffettività del modo di tutela può risolversi nella violazione della norma costituzionale, in quanto derivi direttamente dalla legge così come formulata e strutturata e non dalle modalità, più o meno efficaci della sua applicazione". Né la violazione potrebbe sussistere, a parere della Corte, per l'eventuale ritardo che potrebbe registrare l'azione innanzi all'Autorità Giudiziaria, in considerazione del fatto che l'art. 24 non garantisce l'immediatezza dell'azione, essendo possibili oneri ex lege finalizzati alla tutela di interessi superiori.

Superato l'ostacolo dell'incostituzionalità, è comunque indicativo che laddove c'era, cioè nei rapporti di lavoro subordinato, la conciliazione obbligatoria è fallita, riducendosi a mero e vuoto rituale, al quale le parti il piú delle volte neppure si presentavano. ${ }^{10}$

Sta di fatto che nel 2009 si è inteso dare attuazione alla direttiva dell'UE 2008/52/CE dando delega al Governo affinché provvedesse ad introdurre e disciplinare la mediazione e conciliazione ${ }^{11}$; assunto attuato con il successivo decreto legislativo del 4 marzo 2010, n. $28 .^{12}$

Il risultato mi sembra pieno di contraddizioni e incongruenze.

Innanzitutto la mediazione è stata prevista non per il suo valore intrinseco di strumento piú duttile e maggiormente idoneo a recepire le istanze dei contendenti e, 
in generale, degli uomini, bensí per la sua presunta capacità di far diminuire il carico, davvero eccessivo e destinato a tempi quasi biblici, dei processi italiani.

Pia illusione, come la citata esperienza del contenzioso del lavoro ha già ampiamente dimostrato.

La direttiva europea e lo spirito della mediazione sono stati traditi dalla normativa italiana. Infatti, mentre la direttiva, pur nella sua ottica ristretta al contenzioso giudiziario, ipotizza una mediazione a tutto campo, in Italia, proprio per la predetta finalità pre-processuale (tendente a ridurre i processi civili), la mediazione è poco libera, essendo imposta in via obbligatoria, ed ha margini ristretti, poiché interviene solo sulla stessa materia dell'eventuale futura $\operatorname{lite}^{13} \mathrm{Di}$ conseguenza, viene meno allo scopo principale: quello di consentire una valutazione che travalichi gli aspetti di legge, per esperire accomodamenti di reciproco gradimento. Ad esempio, "un litigante ... potrebbe rinunciare ad una parte del credito o del diritto perché in cambio gli viene offerto un nuovo contratto, o nuove opportunità di lavoro o guadagno, od un diverso bene che egualmente ha interesse a ricevere, ecc... Tutto questo, però, il mediatore del d. lgs. 28/10 non lo può proporre, e quindi nessuna conciliazione di questo genere può essere immaginata con l'ausilio del mediatore. ... il mediatore può fare solo proposte che stiano nell'ambito dell'oggetto della disputa, e tali da potere essere poi accolte o rigettate dal giudice, poiché, se ne facesse altre, diversificando le proposte di conciliazione oltre i limiti dell'oggetto del processo, il meccanismo delle spese salterebbe, e con esso anche la struttura e le finalità del d. lgs. 28/10. Cosí, il d. lgs. 28/10, invece di attribuire al mediatore ogni più ampia libertà nell'individuare l'oggetto della possibile conciliazione, gli impone di muoversi entro il tracciato delle domande delle parti, limitando la sua funzione e pregiudicando in molti casi l'obiettivo che il sistema invece dovrebbe avere, e che è quello di giungere alla transazione della lite". ${ }^{14}$

Se a ciò si aggiunge l'evidente strumentalizzazione della mediazione-conciliazione a favore degli avvocati, i quali, ragionando proprio sulla natura pre-processuale dell'innovazione sostengono di dovere essere sempre presenti, mentre i loro gli ordini professionali sono stati riconosciuti come centri per la conciliazione, a discapito evidente della terzietà e imparzialità dell'esercizio dell'avvocatura. Perché, poi, una grave e pericolosa anomalia della normativa italiana è stata quella di prevedere che la conciliazione non sia effettuata direttamente dai mediatori-conciliatori abilitati e riconosciuti come tali dal Ministero della Giustizia, bensí da appositi centri, i quali diventano centri di affare e arbitri della scelta dei mediatori.

Insomma vi sono aspetti che gettano un'ombra di dubbio e sospetto sulle finalità reali perseguibili con la mediazione e in concreto scaturenti dalla normativa italiana. ${ }^{15}$ 
Tra avvocati sul piede di guerra, per combattere la conciliazione obbligatoria e per far affermare la necessità della propria presenza, e gente comune, fiduciosa di poter esperire un meccanismo non costoso, rapido e in grado di andare di là dalla ragione meramente formale ancorché legale, occorre rimeditare in termini piú generali sulla mediazione. A partire dalla considerazione che essa abbraccia tutti gli aspetti della vita odierna, nella quale ha sempre mena incidenza il discrimine tra pubblico e privato, sicché la mediazione ha ragione di essere invocata e di potere risultare utile in molti campi, ivi compresi quelli pubblici, dai quali sempre di piú in misura maggiore dipendono anche gli interessi dei singoli.

L'esclusione della materia amministrativa nella direttiva europea si giustifica non perché non possa essere oggetto di mediazione, bensí perché anzi richiede una mediazione piú continua e penetrante. La quale (questo sí) non può assolutamente essere legata o proiettata al processo. E vorrei qui ricordare che proprio in quello che potremmo indicare come 'pubblico' la mediazione si è affermata per prima ed in termini piú generali, che abbracciano l'ampia gamma dei rapporti tra cittadini e diritto.

\section{Gli Ombudsman}

Il fenomeno della mediazione e dei 'mediatori' nel pubblico non può essere ritenuto materia a sé ed ininfluente sulla mediazione privata; esso invece concorre all'obiettivo essenziale di garantire all'uomo l'accesso alla giustizia, in ogni aspetto dell'esperienza contemporanea.

Di conseguenza, mi sembra opportuno richiamare (per apices) alcuni tratti della mediazione 'pubblica'.

Nel pubblico pare inarrestabile la creazione di 'mediatori', variamente denominati: ombudsman, mediateur, parliamentary commissioner, defensores del pueblo, avvocati del popolo, ecc. ${ }^{16}$ Alla base di essi vi è il desiderio di dare agli uomini la possibilità di avere un organo in grado di operare come 'intermediario' tra i cittadini, sia tra loro sia nei confronti degli organi di Governo, in modo da comporre le discriminazioni e le recriminazioni, attraverso organi che abbiano maggiori possibilità di incidenza rispetto a quelle che potrebbe mai avere il singolo.

La mediazione che ne scaturisce si differenzia dalle forme di controllo di legittimità e/o di merito degli atti della pubblica amministrazione e cerca di conciliare 'a monte' le aspettative degli uomini con le azioni della p.a., da un lato con lo scopo di prevenire le lungaggini e le spese dei 'ricorsi' alla 'Giustizia', amministrativa e civile, dall'altro per consentire di rappresentare le proprie esigenze liberamente e di là dal mero controllo formale e/o discrezionale. 
Le articolazioni assunte dalla mediazione nel 'pubblico' sono varie e, secondo alcuni, richiamano le gloriose istituzioni dell'esperienza giuridica romana, quali il Tribunato della plebe e i Defensores civitatum. ${ }^{17}$

Esse costituiscono uno strumento di controllo ma anche un tramite tra i singoli e i detentori del potere ed hanno animato ed innervato il dibattito che ha accompagnato la nascita dello Stato contemporaneo, da Pagano al Rouzier, da Montesquieu a Rousseau, da Altusio a Fichte, quando si discusse della necessità di creare organi che, sull'esempio dell'Eforato dell'antica Sparta o dei Tribuni plebis fossero in grado di garantire concretamente la sovranità al popolo. ${ }^{18}$

Oggi queste figure potrebbero svolgere una penetrante opera d'intermediazione ed assumere iniziative a favore degli interessi dei singoli, che dovrebbero essere riconsiderate attentamente assieme alla discussione che concerne i mediatori-conciliatori finalizzati al processo. ${ }^{19}$

\section{Verso un nuovo processo civile}

Collaterale alle proposte che sono state avanzate e talora pretese, perché ritenute addirittura prova della democraticità degli Stati e dirette alla tutela dell'uomo ${ }^{20}$, mi sembra la riconsiderazione del processo civile, con la limitazione dell'ingerenza dello Stato (attuato attraverso i giudici dell'ordinamento giudiziario statale). La critica dell'attuale assetto del processo civile in direzione della restituzione ai privati dei loro poteri di disporre degli interessi in conflitto, appare meno approfondita nella storia del pensiero contemporaneo; tuttavia, a ben vedere, essa prospetta le stesse istanze di difesa della sovranità (di base) dei singoli, che sono invocate a fondamento della mediazione 'pubblica'. Invero, spesso si assiste all'ingerenza immotivata dello Stato contemporaneo, nel processo civile; la quale si traduce in una sottrazione ai privati della disponibilità dei propri interessi ed, in ultima istanza, nella sottrazione di quella sovranità che dovrebbe appartenere al popolo ed essere esercitata da esso, quando non sia in discussione il bene comune.

Qui intendo sottolineare l'urgenza di ridare agli uomini l'esercizio ed il potere di decidere da soli dei propri interessi, avendo presente che anche ai fini della realizzazione del dettato costituzionale degli art. 24 e 3, occorre ridare snellezza, semplicità, velocità e, possibilmente, gratuità all'accesso alla Giustizia per le vie normali, che sono quelle del processo, civile ed amministrativo. In ciò lasciando uno spazio per la mediazione, ma non come forma di pre-processo, bensí come forma differente di concepire il riassetto degli equilibri turbati, vuoi dall'inadempimento di una parte vuoi persino dall'uso di strumenti legali, che possano configurare atti legittimi, ma ugualmente 'ingiusti'. 
È in questo piú ampio spazio che va collocata la mediazione, la quale potrà ristabilire l'equità e proporsi sia in previsione di liti sia in difesa di ragioni dei deboli, oppure per elaborare soluzioni innovative che ridefiniscano i rapporti tra le parti e tra i cittadini ed il potere (pubblico e/o privato), in direzione della giustizia sostanziale e del diritto capace di andare oltre la legge.

Note:

1. Ne trascrivo il testo. Articolo 1. Obiettivo e ambito di applicazione. 1. La presente direttiva ha l'obiettivo di facilitare l'accesso alla risoluzione alternativa delle controversie e di promuovere la composizione amichevole delle medesime incoraggiando il ricorso alla mediazione e garantendo un'equilibrata relazione tra mediazione e procedimento giudiziario. 2. La presente direttiva si applica, nelle controversie transfrontaliere, in materia civile e commerciale tranne per i diritti e gli obblighi non riconosciuti alle parti dalla pertinente legge applicabile. Essa non si estende, in particolare, alla materia fiscale, doganale e amministrativa né alla responsabilità dello Stato per atti o omissioni nell'esercizio di pubblici poteri (acta iure imperii). 3. Nella presente direttiva per "Stato membro" si intendono gli Stati membri ad eccezione della Danimarca. Articolo 2 Controversie transfrontaliere. 1. Ai fini della presente direttiva per controversia transfrontaliera si intende una controversia in cui almeno una delle parti è domiciliata o risiede abitualmente in uno Stato membro diverso da quello di qualsiasi altra parte alla data in cui: a) le parti concordano di ricorrere alla mediazione dopo il sorgere della controversia; b) il ricorso alla mediazione è ordinato da un organo giurisdizionale; c) l'obbligo di ricorrere alla mediazione sorge a norma del diritto nazionale; o d) ai fini dell'articolo 5, un invito è rivolto alle parti. 2. In deroga al paragrafo 1, ai fini degli articoli 7 e 8 per controversia transfrontaliera si intende altresi una controversia in cui un procedimento giudiziario $o$ di arbitrato risultante da una mediazione tra le parti è avviato in uno Stato membro diverso da quello in cui le parti erano domiciliate o risiedevano abitualmente alla data di cui al paragrafo 1 , lettere a), b) o c). 3 . Ai fini dei paragrafi 1 e 2 , il domicilio è stabilito in conformità degli articoli 59 e 60 del regolamento (CE) n. 44/2001. Articolo 3 Definizioni. Ai fini della presente direttiva si applicano le seguenti definizioni: a) per "mediazione" si intende un procedimento strutturato, indipendentemente dalla denominazione, dove due o più parti di una controversia tentano esse stesse, su base volontaria, di raggiungere un accordo sulla risoluzione della medesima con l'assistenza di un mediatore. Tale procedimento può essere avviato dalle parti, suggerito od ordinato da un organo giurisdizionale o prescritto dal diritto di uno Stato membro. Esso include la mediazione condotta da un giudice che non è responsabile di alcun procedimento giudiziario concernente la controversia in questione. Esso esclude i tentativi messi in atto dall'organo giurisdizionale $o$ dal giudice aditi al fine di giungere ad una composizione della controversia in questione nell'ambito del procedimento giudiziario oggetto della medesima; b) per "mediatore" si intende qualunque terzo cui è chiesto di condurre la mediazione in modo efficace, imparziale e competente, indipendentemente dalla denominazione o dalla professione di questo terzo nello Stato membro interessato e dalle modalità con cui è stato nominato o invitato a condurre la mediazione. Articolo 4 Qualità della mediazione. 1. Gli Stati membri incoraggiano in qualsiasi modo da essi ritenuto appropriato l'elaborazione di codici volontari di condotta da parte dei mediatori e delle organizzazioni che forniscono servizi di mediazione nonché l'ottemperanza ai medesimi, cosi come qualunque altro efficace meccanismo di controllo della qualità riguardante la fornitura di servizi di mediazione. 2. Gli Stati membri incoraggiano la formazione iniziale e successiva dei mediatori allo scopo di garantire che la mediazione sia gestita in maniera efficace, imparziale e competente in relazione alle parti. Articolo 5 Ricorso alla mediazione. 1. L'organo giurisdizionale investito di una causa può, se lo ritiene appropriato e tenuto conto di tutte le circostanze del caso, invitare le parti a ricorrere alla mediazione allo scopo di dirimere la controversia. Può altresì invitare le parti a partecipare ad una sessione informativa sul ricorso alla mediazione se tali sessioni hanno luogo e sono facilmente accessibili. 2. La presente direttiva lascia impregiudicata la legislazione nazionale che rende il ricorso alla mediazione obbligatorio oppure soggetto a incentivi o sanzioni, sia prima che dopo l'inizio del procedimento giudiziario, purché tale legislazione non impedisca alle parti di esercitare il diritto di accesso al sistema giudiziario. Articolo 6 Esecutività degli accordi risultanti dalla mediazione 1. Gli Stati membri assicurano che le parti, o una di esse con l'esplicito consenso delle altre, abbiano la possibilità di chiedere che il contenuto di un accordo scritto risultante da una mediazione sia reso esecutivo. II contenuto di tale accordo è reso esecutivo salvo se, nel caso in questione, il contenuto dell'accordo è contrario alla legge dello Stato membro in cui viene presentata la richiesta o se la legge di detto Stato membro non ne prevede l'esecutività. 2. II contenuto dell' accordo può essere reso esecutivo in una sentenza, in una decisione o in un atto autentico da un organo giurisdizionale 0 da un'altra autorità competente in conformità del diritto dello Stato membro in cui è presentata la richiesta. 3. Gli Stati membri indicano alla Commissione gli organi giurisdizionali o le altre autorità competenti a ricevere le richieste conformemente ai paragrafi 1 e 2. 4. Nessuna disposizione del presente articolo pregiudica le norme applicabili al riconoscimento e all'esecuzione in un altro Stato membro di un accordo reso esecutivo in conformità del paragrafo 1. Articolo 7 Riservatezza della mediazione. 1. Poiché la mediazione deve avere luogo in modo da rispettare la riservatezza, gli Stati membri garantiscono che, a meno che le parti non decidano diversamente, né i mediatori né i soggetti coinvolti nell'amministrazione del procedimento di 
mediazione siano obbligati a testimoniare nel procedimento giudiziario o di arbitrato in materia civile e commerciale riguardo alle informazioni risultanti da un procedimento di mediazione o connesse con lo stesso, tranne nei casi in cui: a) ciò sia necessario per superiori considerazioni di ordine pubblico dello Stato membro interessato, in particolare sia necessario per assicurare la protezione degli interessi superiori dei minori o per scongiurare un danno all'integrità fisica o psicologica di una persona; oppure b) la comunicazione del contenuto dell'accordo risultante dalla mediazione sia necessaria ai fini dell'applicazione o dell'esecuzione di tale accordo. 2. II paragrafo 1 non impedisce in alcun modo agli Stati membri di adottare misure più restrittive per tutelare la riservatezza della mediazione. Articolo 8 Effetto della mediazione sui termini di prescrizione e decadenza. 1. Gli Stati membri provvedono affinché alle parti che scelgono la mediazione nel tentativo di dirimere una controversia non sia successivamente impedito di avviare un procedimento giudiziario o di arbitrato in relazione a tale controversia per il fatto che durante il procedimento di mediazione siano scaduti i termini di prescrizione o decadenza. 2. II paragrafo 1 lascia impregiudicate le disposizioni relative ai termini di prescrizione o decadenza previste dagli accordi internazionali di cui gli Stati membri sono parte. Articolo 9 Informazioni al pubblico. Gli Stati membri incoraggiano, in qualsiasi modo ritengano appropriato, la divulgazione al pubblico, in particolare via Internet, di informazioni sulle modalità per contattare i mediatori e le organizzazioni che forniscono servizi di mediazione. Articolo 10 Informazioni sugli organi giurisdizionali e sulle autorità competenti. La Commissione mette a disposizione del pubblico, tramite qualsiasi mezzo appropriato, le informazioni sugli organi giurisdizionali o sulle autorità competenti comunicate dagli Stati membri ai sensi dell'articolo 6, paragrafo 3. Articolo 11 Revisione. Entro il 21 maggio 2016 la Commissione presenta al Parlamento europeo, al Consiglio e al Comitato economico e sociale europeo una relazione sull'attuazione della presente direttiva. La relazione esamina lo sviluppo della mediazione nell'Unione europea e l'impatto della presente direttiva negli Stati membri. Se del caso, la relazione è corredata di proposte di modifica della presente direttiva. Articolo 12 Attuazione. 1. Gli Stati membri mettono in vigore le disposizioni legislative, regolamentari e amministrative necessarie per conformarsi alla presente direttiva anteriormente al 21 maggio 2011, fatta eccezione per l'articolo 10, per il quale tale data è fissata al più tardi al 21 novembre 2010. Essi ne informano immediatamente la Commissione. Quando gli Stati membri adottano tali disposizioni, queste contengono un riferimento alla presente direttiva o sono corredate di un siffatto riferimento all'atto della pubblicazione ufficiale. Le modalità di tale riferimento sono decise dagli Stati membri. 2. Gli Stati membri comunicano alla Commissione il testo delle principali disposizioni di diritto interno che essi adottano nel settore disciplinato dalla presente direttiva. Articolo 13 Entrata in vigore. La presente direttiva entra in vigore il ventesimo giorno successivo alla pubblicazione nella Gazzetta ufficiale dell'Unione europea. Articolo 14 Destinatari. Gli Stati membri sono destinatari della presente direttiva.

2. Capo VI. Giustizia. Articolo 47. Diritto a un ricorso effettivo e a un giudice imparziale. Ogni individuo i cui diritti e le cui libertà garantiti dal diritto dell'Unione siano stati violati ha diritto a un ricorso effettivo dinanzi a un giudice, nel rispetto delle condizioni previste nel presente articolo. Ogni individuo ha diritto a che la sua causa sia esaminata equamente, pubblicamente ed entro un termine ragionevole da un giudice indipendente e imparziale, precostituito per legge. Ogni individuo ha la facoltà di farsi consigliare, difendere e rappresentare. A coloro che non dispongono di mezzi sufficienti è concesso il patrocinio a spese dello Stato qualora ciò sia necessario per assicurare un accesso effettivo alla giustizia. Articolo 48. Presunzione di innocenza e diritti della difesa. 1. Ogni imputato è considerato innocente fino a quando la sua colpevolezza non sia stata legalmente provata. 2. II rispetto dei diritti della difesa è garantito ad ogni imputato. Articolo 49. Principi della lega lità e della proporzionalità dei reati e delle pene. 1. Nessuno può essere condannato per un'azione o un'omissione che, al momento in cui è stata commessa, non costituiva reato secondo il diritto interno o il diritto internazionale. Parimenti, non può essere inflitta una pena più grave di quella applicabile al momento in cui il reato è stato commesso. Se, successivamente alla commissione del reato, la legge prevede l'applicazione di una pena più lieve, occorre applicare quest'ultima. 2. II presente articolo non osta al giudizio e alla condanna di una persona colpevole di un'azione o di un'omissione che, al momento in cui è stata commessa, costituiva un crimine secondo i principi generali riconosciuti da tutte le nazioni. 3. Le pene inflitte non devono essere sproporzionate rispetto al reato. Articolo 50 . Diritto di non essere giudicato o punito due volte per lo stesso reato. Nessuno può essere perseguito o condannato per un reato per il quale è già stato assolto o condannato nell'Unione a seguito di una sentenza penale definitiva conformemente alla legge.

3. In casi sempre piú numerosi il commercio ed i contratti investono privati e grandi imprese ed è ovvio che queste ultime dispongono di avvocati e di mezzi molto superiori ai privati, avendo per ciò stesso maggiori probabilità di prevalere davanti ai Tribunali.

4. Cfr., M. MARINARI, L'esperienza dell'ADR negli Stati uniti ed in Inghilterra, in Documenti Giustizia 2000. Infatti, anche se si è affermato che la 'culla delle forme alternative sia stata l'Oriente ed in particolare la Cina propensa per sua cultura alla composizione amichevole della lite, è negli U.S.A. che va collocata la nascita della mediazione come strumento di risoluzione dei conflitti, databile intorno al 1906 quando nacque un movimento culturale delle Alternative Dispute ovvero di metodi di risoluzione alternativa delle dispute (ADR) Questo movimento in una conferenza di studi, celebrata in onore di Roscoe Pound, tenutasi dinnanzi all'American Bar Association, aveva denunciato gravi disfunzioni del sistema di amministrazione della giustizia che avevano causato la litigation explosion sino alla litigation crisis Resolution, prospettando l'opportunità di sistemi alternativi di risoluzione dei conflitti giudiziari. Nel 1983 attraverso un emendamento al Federal Rules of Civil Procedure (il Codice federale di procedura civile americano), si previde che nel corso della pretrial conference (quando l'autorità giudiziaria delinea l'oggetto della causa) il giudice aveva l'obbligo di tentare una transazione (settlement) illustrando alle parti la 
possibilità di ricorrere anche a procedure extragiudiziali o endoprocessuali di arbitrato obbligatorio o di strumenti alternativi al giudizio ordinario, in via sperimentale e con la supervisione da parte delle corti degli ADR.(court annexed programs e ADR court connected programs). Nel 1990 l'approvazione del Civil Justice Reform Act da parte del Congresso suggellava l'utilizzo dei metodi ADR e della mediation. Nel 1998, attraverso l'Alternative Dispute Resolution Act, si pose fine alla fase sperimentale e si stabilí che ciascuna Corte distrettuale degli Stati Uniti avrebbe potuto autorizzare l'uso di procedure di risoluzione alternative delle controversie in ogni azione civile. Infine nel 2001 la mediation ricevette l'assetto definitivo, attraverso I'Uniform Mediation Act. Le procedure ADR negli Stati Uniti d'America si possono classificare secondo le seguenti tipologie: conciliation - mediation; arbitration - adjudication; settlement conference giudiziaria; court annexed arbitration e court annexed mediation. II primo tipo configura un modello conciliativo - mediativo che mira alla risoluzione della controversia tra le parti mediante l'accordo delle stesse facilitato dall'intervento di mediazione di un terzo che non emette una decisione vincolante. Tale modello va distinto dalla negotiation in cui avviene una contrattazione diretta tra le parti. Il secondo rimane un modello aggiudicativo ove il terzo arbitro con una procedura formale e riservata emette un giudizio privato vincolante tra le parti. II terzo modello configura una conciliazione giudiziaria che viene riservata ai giudici nella fase introduttiva del giudizio. La quarta figura è una procedura che ha carattere endoprocessuale, che viene delegata dal giudice a terzi anche su richiesta delle parti. Cfr. G. Rossillo, I mezzi alternativi di risoluzione delle controversie (ADR) tra diritto comunitario e diritto internazionale, in II diritto dell'Unione europea, a. XIII fasc. e 2008.

5. Risale all'Assemblea costituente francese l'istituzione (con Decreto del $16-14$ agosto del $1790 \mathrm{n} .16 \mathrm{nel} 1790$ ) del juge de paix et de conciliation, che era un magistrato laico onorario dotato di funzioni giurisdizionali, di giurisdizione volontaria e di funzioni di conciliazione. Dapprima era elettivo (da parte delle assemblee primarie), poi diventò di nomina governativa fu successivamente nominato dal governo. II ricorso al juge de paix et de conciliation era obbligatorio e preventivo, non potendosi procedere all'adizione di un giudice ordinario senza documentare di avere prima esperita la conciliazione. Tale obbligatorietà della conciliazione preventiva fu mantenuta dal Code Napoléon del 1806, attraverso l'art. 48, del libro II titolo I, anche se, a parere unanime, essa era di fatto vanificata dal successivo art. 49. La Giurisprudenza considerò l'obbligatorietà in un modo particolare, la ritenne, cioè, un motivo di invalidità della successiva procedura, rilevabile esclusivamente su istanza del convenuto. Si determinò, in realtà, un clima di sfiducia verso la conciliazione, motivato in gran parte dal fatto che il juge de paix et de conciliation era pur sempre un 'giudice, con funzioni conciliative ma anche giurisdizionali, assumente un posizione di predominanza che mal si accordavano con il ruolo di parità necessarie in un pacificator-mediatore. In tempi recenziori la conciliazione registrò un ritorno di fiamma, che portò alla previsione di un conciliateur de justice previsto nel Decret n. 381-78 del 20 mar.1978. Esso è un organo extragiudiziale, privo di poteri giurisdizionali, che ha il compito de faciliter bevevolment ed in via amichevole il contenzioso al fine di favorire la résolution des litiges. Secondo la dottrina francese si è aperta la strada a Modes Alternatifs de Réglement des Conflits (M.A.R.C.) con caratteristiche e radici indipendenti dai modelli delle Alternative Dispute Resolution. La competenza du conciliateur de justice verte sui soli diritti disponibili rimanendo escluse dalla sua competenza tutte le questioni in tema di stato, capacità delle persone, divorzio, separazione dei coniugi e le materie di ordine pubblico che sono riservate al giudice ordinario. Va però notato che nel frattempo si era registrata la nascita del Médiateur de la République de France che, per certi versi, completava il quadro della mediazione, per le questioni che, direttamente 0 indirettamente, dipendevano dalla p.a. inoltre in ámbiti specifici sono emerse nuove figure: come le conciliateur médical e le conciliateur de la consommation. In generale les concilateurs sono sfornite di potere coercitivo sulle parti o decisionale. Queste sono tutte forme di conciliazione stragiudiziaria. L'ordinamento francese conosce anche la conciliazione giudiziaria, attuata, con il consenso delle parti, da un delegato del giudice 0 , in caso di mancata richiesta delle parti, direttamente dal giudice della causa. Inoltre, presso il Tribunal de grande instance di Parigi, con esclusione dalla Corte di Cassazione, è stata introdotta la mediation judiciaire, esperita, sempre nell'ámbito dei diritti disponibili, su rilevanti questioni di diritto, attraverso una proposta di accordo (constat d'accord) che le partisono libere di accettare o meno. Quest'ultimo approdo della mediazione in Francia si avvicina all ADR di matrice anglosassone. Punto di forza della mediazione francese è la brevità: massimo 3 mesi. Cfr., tra i tanti, L. CADIET, I modi alternativi di regolamento dei conflitti in Francia tra tradizione e modernità, in Riv. trim. dir. e proc. civ. 2006; G. ROSSILLO, I mezzi alternativi di risoluzione delle controversie (ADR) tra diritto comunitario e diritto internazionale cit.

6. Cfr. M. KASER, Das römisches Zivilprozessrecht, München 1996, cfr. particolarmente l'indice, alle voci arbiter e compromissum.

7. Cosí M. MARRONE, Sull'arbitrato privato nell'esperienza giuridica romana, in Rivista dell'arbitrato 1, 1996, p. 1. L'a. pone in evidenza la permanenza dell'arbitrato per tutto l'arco del diritto romano e ne individua le ragioni nella maggiore snellezza, nella gratuità e nella immediatezza: “... il procedimento relativo era gratuito ... e, nonostante i tanti interventi legislativi, il dibattito arbitrale era assai più snello e semplice rispetto al processo ordinario, e ampia rimase complessivamente l'autonomia lasciata ai contendenti; ai quali, d'altronde, erano assicurate ... maggiori garanzie" (p. 16, concernente l'età postclassica e giustinianea).

8. M. MARRONE, op. cit., p. 17.

9. V.M. Marrone, op. cit., p. 2. M. TALAMANCA, Istituzioni di diritto romano, Milano 1990, p. 568 s.

10. Ricordo che l'art. 410 c.p.c. dispone che: Chi intende proporre in giudizio una domanda relativa ai rapporti previsti dall'articolo 409, e non ritiene di avvalersi delle procedure di conciliazione previste dai contratti e accordi collet- 
tivi deve promuovere, anche tramite l'associazione sindacale alla quale aderisce o conferisce mandato, il tentativo di conciliazione, presso la commissione di conciliazione individuata secondo i criteri di cui all'articolo 413 c.p.c. II successivo art. 411 c.p.c. stabilisce che, se la conciliazione non riesce, si redige processo verbale firmato dal presidente del collegio che certifica l'autenticità della sottoscrizione o l'impossibilità a sottoscrivere delle par$\mathrm{ti}$, per lo piú perché assenti. Per le controversie individuali e plurime, la legge prevede il tentativo obbligatorio di conciliazione, configurandolo come condizione di procedibilità per la successiva domanda giudiziaria (art. 412: l'espletamento del tentativo di conciliazione costituisce condizione di procedibilità della domanda), la cui mancanza deve essere rilevata dal giudice adito anche d'ufficio, ma non comporta la caduta della causa, bensí la sospensione del giudizio e l'assegnazione di un termine alle parti per il relativo espletamento. Successivamente, con l'art. del d.Ig. 31.3.1998, n. 80 è stata introdotta l'obbligatorietà del tentativo di conciliazione extragiudiziale. In conseguenza, in materia di controversie di lavoro si può procedere o attraverso la conciliazione sindacale, avvalendosi delle procedure di conciliazione eventualmente previste dai contratti o dagli accordi collettivi (art. 411 c.p.c.), oppure una conciliazione amministrativa, non intendendo avvalersi delle procedure sopra menzionate, ma ricorrendo, anche tramite l'associazione sindacale, a specifica Commissione di conciliazione presso la Direzione provinciale del lavoro (art. 410 c.p.c.). L'art. 410 bis, $1^{\circ}$ co., c.p.c. prevede poi che: il tentativo di conciliazione, anche se nelle forme previste dai contratti o accordi collettivi, deve essere espletato entro 60 giorni dalla presentazione della richiesta e trascorso inutilmente tale termine il tentativo di conciliazione si considera comunque espletato ai fini dell'art. 412 bis cod. proc. civ. (così il $2^{\circ}$ co. dell'art. 412 bis c.p.c.).

11. L. 19 giugno 2009, n. 69 Disposizioni per lo sviluppo economico, la semplificazione, la competitività nonché in materia di processo civile. Art. 60 Delega al Governo in materia di mediazione e di conciliazione delle controversie civili e commerciali della legge: 1. II Governo è delegato ad adottare, entro sei mesi dalla data di entrata in vigore della presente legge, uno o piú decreti legislativi in materia di mediazione e di conciliazione in ambito civile e commerciale. 2. La riforma adottata ai sensi del comma 1, nel rispetto e in coerenza con la normativa comunitaria e in conformità ai principi e criteri direttivi di cui al comma 3 , realizza il necessario coordinamento con le altre disposizioni vigenti. I decreti legislativi previsti dal comma 1 sono adottati su proposta del Ministro della giustizia e successivamente trasmessi alle Camere, ai fini dell'espressione dei pareri da parte delle Commissioni parlamentari competenti per materia e per le conseguenze di carattere finanziario, che sono resi entro il termine di trenta giorni dalla data di trasmissione, decorso il quale i decreti sono emanati anche in mancanza dei pareri. Qualora detto termine venga a scadere nei trenta giorni antecedenti allo spirare del termine previsto dal comma 1 o successivamente, la scadenza di quest'ultimo è prorogata di sessanta giorni. 3. Nell'esercizio della delega di cui al comma 1, il Governo si attiene ai seguenti principi e criteri direttivi: a) prevedere che la mediazione, finalizzata alla conciliazione, abbia per oggetto controversie su diritti disponibili, senza precludere l'accesso alla giustizia; b) prevedere che la mediazione sia svolta da organismi professionali e indipendenti, stabilmente destinati all'erogazione del servizio di conciliazione; c) disciplinare la mediazione, nel rispetto della normativa comunitaria, anche attraverso l'estensione delle disposizioni di cui al decreto legislativo 17 gennaio 2003, n. 5, e in ogni caso attraverso l'istituzione, presso il Ministero della giustizia, senza nuovi o maggiori oneri per la finanza pubblica, di un Registro degli organismi di conciliazione, di seguito denominato "Registro", vigilati dal medesimo Ministero, fermo restando il diritto delle camere di commercio, industria, artigianato e agricoltura che hanno costituito organismi di conciliazione ai sensi dell'art. 2 della legge 29 dicembre 1993, n. 580, ad ottenere l'iscrizione di tali organismi nel medesimo Registro; d) prevedere che i requisiti per l'iscrizione nel Registro e per la sua conservazione siano stabiliti con decreto del Ministro della giustizia; e) prevedere la possibilità, per i consigli degli ordini degli avvocati, di istituire, presso i tribunali, organismi di conciliazione che, per il loro funzionamento, si avvalgono del personale degli stessi consigli; f) prevedere che gli organismi di conciliazione istituiti presso i tribunali siano iscritti di diritto nel Registro; g) prevedere, per le controversie in particolari materie, la facoltà di istituire organismi di conciliazione presso i consigli degli ordini professionali; h) prevedere che gli organismi di conciliazione di cui alla lettera g) siano iscritti di diritto nel Registro; i) prevedere che gli organismi di conciliazione iscritti nel Registro possano svolgere il servizio di mediazione anche attraverso procedure telematiche; I) per le controversie in particolari materie, prevedere la facoltà del conciliatore di avvalersi di esperti, iscritti nell'albo dei consulenti e dei periti presso i tribunali, i cui compensi sono previsti dai decreti legislativi attuativi della delega di cui al comma 1 anche con riferimento a quelli stabiliti per le consulenze e per le perizie giudiziali; $\mathrm{m}$ ) prevedere che le indennità spettanti ai conciliatori, da porre a carico delle parti, siano stabilite, anche con atto regolamentare, in misura maggiore per il caso in cui sia stata raggiunta la conciliazione tra le parti; n) prevedere il dovere dell'avvocato di informare il cliente, prima dell'instaurazione del giudizio, della possibilità di avvalersi dell'istituto della conciliazione nonché di ricorrere agli organismi di conciliazione; o) prevedere, a favore delle parti, forme di agevolazione di carattere fiscale, assicurando, al contempo, l'invarianza del gettito attraverso gli introiti derivanti al Ministero della giustizia, a decorrere dall'anno precedente l'introduzione della norma e successivamente con cadenza annuale, dal Fondo unico giustizia di cui all'art. 2 del decreto-legge 16 settembre 2008, n. 143, convertito, con modificazioni, dalla legge 13 novembre $2008, n$. 181 ; p) prevedere, nei casi in cui il provvedimento che chiude il processo corrisponda interamente al contenuto dell'accordo proposto in sede di procedimento di conciliazione, che il giudice possa escludere la ripetizione delle spese sostenute dal vincitore che ha rifiutato l'accordo successivamente alla proposta dello stesso, condannandolo altresí, e nella stessa misura, al rimborso delle spese sostenute dal soccombente, salvo quanto previsto dagli articoli 92 e 96 del codice di procedura civile, e, inoltre, che possa condannare il vincitore al pagamento di un'ulteriore somma a titolo di contributo unificato ai sensi dell' art. 9 del testo unico delle disposizioni legislative e regolamentari in materia di spese di giustizia, di cui al decreto del Presiden- 
te della Repubblica 30 maggio 2002, n. 115; q) prevedere che il procedimento di conciliazione non possa avere una durata eccedente i quattro mesi; r) prevedere, nel rispetto del codice deontologico, un regime di incompatibilità tale da garantire la neutralità, l'indipendenza e l'imparzialità del conciliatore nello svolgimento delle sue funzioni; s) prevedere che il verbale di conciliazione abbia efficacia esecutiva per l'espropriazione forzata, per l'esecuzione in forma specifica e costituisca titolo per l'iscrizione di ipoteca giudiziale.». - La direttiva 2008/52/CE è pubblicata nella Gazzetta Ufficiale dell'Unione europea - serie L 136/3 del 24 maggio 2008. Note all'art. 1: - II decreto del Ministro della giustizia 23 luglio 2004, n. 222 reca: «Regolamento recante la determinazione dei criteri e delle modalità di iscrizione nonché di tenuta del registro degli organismi di conciliazione di cui all'art. 38 del D.Lgs. 17 gennaio 2003, n. $5 . »$.

12. Decreto Legislativo 4 marzo 2010, n. 28. Attuazione dell'articolo 60 della legge 18 giugno 2009, n. 69, in materia di mediazione finalizzata alla conciliazione delle controversie civili e commerciali: ॥ Presidente della Repubblica omissis: $\mathrm{E} m$ a $\mathrm{n}$ a il seguente decreto legislativo: Art. 1 Definizioni 1 . Ai fini del presente decreto legislativo, si intende per: a) mediazione: l'attività, comunque denominata, svolta da un terzo imparziale e finalizzata ad assistere due o più soggetti sia nella ricerca di un accordo amichevole per la composizione di una controversia, sia nella formulazione di una proposta per la risoluzione della stessa; b) mediatore: la persona o le persone fisiche che, individualmente o collegialmente, svolgono la mediazione rimanendo prive, in ogni caso, del potere di rendere giudizi o decisioni vincolanti per i destinatari del servizio medesimo; c) conciliazione: la composizione di una controversia a seguito dello svolgimento della mediazione; d) organismo: l'ente pubblico o privato, presso il quale può svolgersi il procedimento di mediazione ai sensi del presente decreto; e) registro: il registro degli organismi istituito con decreto del Ministro della giustizia ai sensi dell'articolo 16 del presente decreto, nonché, sino all'emanazione di tale decreto, il registro degli organismi istituito con il decreto del Ministro della giustizia 23 luglio 2004, n. 222.

13. Sul punto v. le caustiche osservazioni di G. SCARSELLI, La nuova mediazione e conciliazione: le cose che non vanno, in Judicium - II processo civile in Italia e in Europa, consultabile online al sito www.judicum.it.

14. Cosí SCARSELLI, loc.cit.

15. Radicale la critica, ad esempio, di SCARSELLI loc. cit.: "questa normativa sembra pensata apposta per accontentare due categorie di soggetti, i magistrati e gli apparati degli ordini professionali. I magistrati, poiché questi sperano, se non proprio in una riduzione delle sentenze da pronunciare, quanto meno, contro il monito di Calamandrei, in una più ampia possibilità di differire e rinviare le cause, e quindi di allontanare nel tempo la pronuncia delle sentenze. Gli apparati degli ordini professionali, poiché essi vedono in questa novità, e non possono non vederlo, una opportunità di lavoro per gli iscritti. Al di là di questo, il resto è incerto. Per venire veramente incontro ai cittadini, e per favorire veramente la conciliazione delle liti attraverso la mediazione, è necessario invece riportarla ai normali criteri già indicati nelle direttive europee, quale fenomeno semplicemente rimesso alla discrezionalità della parte, e senza condizionamento sul diritto costituzionale all'azione, e senza aspetti punitivi appartenenti ad una logica che non può essere approvata. Poiché solo se la mediazione ha queste caratteristiche essa ha concreta possibilità di dare un contributo alla situazione generale del nostro contenzioso civile". II 'monito' di Calamandrei, citato dall'a. in apertura del suo articolo, è il seguente: "Quante comparizioni personali delle parti, quanti tentativi di conciliazione ... sono ordinati dal giudice istruttore unicamente come espedienti per rimandare l'istruttoria di un mese o due, eppur colla certezza che il tentativo di conciliazione non riuscirà". P. CALAMANDREI, II processo come giuoco, in Riv. dir. proc., 1950, I, 37

16. II termine Ombudsman significava l'uomo che fa da tramite. La sua creazione e risale ad un decreto del 1713 di Carlo XII che, per l'appunto, istituí l'Högste Ombudsman. Comunemente si ritiene che la sua definizione si debba attribuire all'art. 96 della Costituzione del regno di Svezia del 1809, il quale previde, accanto al Justitiekansler, un commissario parlamentare (Justitie Ombudsman), scelto "tra persone di comprovata capacità tecnica, imparzialità ed integrità": cfr., S. TAFARO, Le radici. Proposte, in [cur. A. LOIODICE, S. TAFARO E N. SHEHU], L'avvocato Del Popolo Albanese, vol. 2, Torino 2008 (ISBN: 88-348-4259-6)., pp. 3-24; Ibidem N. SHEHU, Dall'högste Ombudsman all'Avvocato del Popolo Albanese, in L'avvocato Del Popolo Albanese, p. 27 s.

17. Sul punto rinvio alle riflessioni avanzate in altre sedi: S. TAFARO, L'eredità dei «tribuni plebis», in [cur. Maria Pia Baccari e Cosimo Cascione] Tradizione romanistica e Costituzione, diretta da Luigi Labruna. (vol. II, pp. 18411880). Collana "Cinquanta anni della Corte costituzionale della Repubblica italiana». Napoli, 2006. ISBN: 88495-1248-1. S. TAFARO, La herencia de los Tribuni Plebis, in DIRITTO@STORIA (riv. on line). N. 7 - Nuova Serie (2008). ISSN: 1825-0300. S. TAFARO, Continuidad de la función tribunicia, en Estudios en homenaje a Mercedes Gayosso y Navarrete, [cur. JOSÉ LUIS CUEVAS GAYOSSO], pp.353-375, Universidad Veracruzana, México, noviembre de 2009. ISBN 978-607-00-1953-1

18. Sul tema rinvio al mio articolo II controllo del potere: ieri ed oggi, in Scritti Panza, Bari 2010

19. Vorrei precisare che oggi attraverso gli Ombudsman si tende a perseguire l'effettivo ed efficace riconoscimento del ruolo del popolo e della conseguente sovranità insopprimibile di ciascun cittadino, cosí come avveniva in Roma attraverso il Tribunato della plebe. In concreto si cerca di vedere nelle nuove figure (piú o meno ispirate all'originario Ombudsman) le istituzioni invocate in sedi internazionali per la difesa dei diritti dell'uomo. I quali sollevano la questione di chi debba proteggerli, soprattutto quando a violarli siano lo stesso Stato o suoi organi, e di chi possa assumerne le ragioni mediandole dinanzi ai poteri dello Stato e/o della grandi imprese. Si ritiene o si propone che a svolgere tale compito possano essere chiamati proprio le figure, comunque denominate, che impropriamente vengono indicate come Ombudsman. Certamente, in ogni caso, l'impegno e l'attività in difesa dei 
diritti umani assume grande rilievo presso tutti i tipi di Defensores e si sostanzia in molteplici interventi: dalla partecipazione ad organismi e commissioni, che definiscono e ridefiniscono continuamente i diritti dell'uomo, alla promozione di incontri (convegni, seminari, ecc.) per sensibilizzare le istituzioni e l'opinione pubblica sulla tutela dei diritti umani. Non si può, però, sottovalutare anche il fatto che gli Ombudsman (di norma) non hanno legittimazione dinanzi alle istanze internazionali create per la tutela dei diritti dell'uomo; generalmente non hanno neanche legittimazione presso i Tribunali nazionali. Per questa ragione, alcuni di essi hanno chiesto di potere intervenire almeno nel delicato momento dell'acquisizione delle prove, invocando l'estensione dell'istituto di matrice anglosassone dell'Amicus Curiae, con facoltà di partecipazione al processo di chi non vi sarebbe legittimato, per carenza di interesse. Di recente è stato previsto dall'art. 74 del Regolamento per l'istituzione del Tribunale Internazionale penale per i crimini della ex Jugoslavia; su di esso, V. PALCHETTI, Amici curiae davanti alla Corte internazionale di giustizia?, in Rivista di diritto internazionale 83, fasc. 4 (2000) 965-991; H. ASCENSIO, L'amicus curiae devant les juridictions internationales, in Revue générale de droit int. public (2001) $924 \mathrm{~s}$. Ciò sarebbe una svolta innovativa nelle procedure ed affiancherebbe felicemente, nell'interesse dei cittadini, la mediazione, come strumenti di accesso alla Giustizia.

20. V. N. SHEHU Dall'högste Ombudsman all'Avvocato del Popolo Albanese cit., p. 42: "La preoccupazione per i diritti dell'uomo è una costante delle Organizzazioni internazionali. Già nel 1946 presso il Consiglio economico e sociale delle Nazioni Unite fu esaminata una proposta tendente ad affidare la tutela e lo sviluppo dei diritti dell'uomo ad apposite istituzioni nazionali. Si innescarono vari processi per i quali fu necessario fare il punto in tempi piú recenti. Tra le vicende che e le iniziative che ne seguirono va segnalata soprattutto quella del giugno del 1990 , quando i Paesi partecipanti alla Conferenza per la Sicurezza e la Cooperazione, riunita in Danimarca a Copenaghen, proposero, in primo luogo agli Stati partecipanti ma in generale ad ogni Paese, di "rendere possibile la creazione e il consolidamento delle istituzioni nazionali, indipendenti nel campo dei diritti dell'uomo e dello Stato del diritto". L'anno dopo furono enunciati i "Principi di Parigi" i quali, a conclusione della Conferenza internazionale, tenutasi a Parigi, tra le istituzioni nazionali che si occupano della difesa e lo sviluppo dei diritti dell'uomo, enunciarono i punti essenziali per fare in modo che le legislazione dei singoli Stati creassero o rafforzassero attraverso la propria legislazione in maniera chiara ed esplicita le istituzioni che dovevano badare alla difesa ed allo sviluppo dei diritti dell'uomo. Questa esigenza fu confermata e sottolineata con forza nel 1993 dalla Dichiarazione di Vienna, la quale tornò a chiedere la creazione ed il rafforzamento di istituzioni nazionali dirette alla salvezza e promozione dei diritti dell'uomo. In conseguenza di queste spinte e della forza che la tutela dei diritti dell'uomo stava assumendo presso l'opinione pubblica mondiale alcuni Paesi pensarono di utilizzare gli istituti dell'Ombudsman per affidare loro la tutela dei diritti dell'uomo" 


\section{MEDIAZIONE E PROCESSO: CRISI DEL PROCESSO E RICORSO ALLA CONCILIAZIONE}

La mediazione nell'UE. - Il nostro tempo assiste alla riscoperta e al rilancio a tutti i livelli sia riguardo al 'pubblico' si riguardo al 'privato' della mediazione.

In particolare si pensa di risolvere le difficoltà di accesso alla giustizia attraverso l'introduzione della mediazione e conciliazione, come forma parallela ẹ stragiudiziale di composizione delle liti.

È questa la soluzione escogitata in sede europea all'interno dell'UE.

L'Unione, infatti, dopo un lungo e faticoso percorso ha emanato una direttiva che si muove in questa direzione e impegna gli Stati membri a introdurre forme di conciliazione spontanea e preventiva delle liti.

Si tratta della direttiva 2008/52/CE del Parlamento europeo e del Consiglio del 21 maggio 2008, entrata in vigore il 12.6.2008, la quale promuove il ricorso alla mediazione come metodo di risoluzione consensuale delle controversie in materia civile e commerciale ed impegna gli Stati membri a realizzarne il contenuto entro maggio 2011 .

La direttiva, comunque, rappresenta un esempio del riconoscimento del diritto alla giustizia come diritto fondamentale e segna un passo avanti rispetto alla Carta dei diritti fondamentali dell'UE del 2000 , nella quale il diritto alla giustizia, previsto al capo VI, era considerato nell'ottica dell'organizzazione tradizionale della giustizia, vale a dire quella dei Tribunali, con attenzione accentuata sulle procedure penali. La nuova direttiva si fonda sulla raggiunta consapevolezza che le difficoltà di accesso alla giustizia in campo civile e commerciale ledono diritti fondamentali e possono essere non meno devastanti di una condanna penale.

L'Ue, con questa direttiva, si fa carico dell'esigenza di assicurare un giudizio di equità a parti che possono avere peso e capacità di organizzarsi nel processo molto differenti.

Il giudizio di equità proposto attraverso la mediazione mi sembra dettato da diverse consapevolezze, dipendenti dai cambiamenti dei sistemi giuridici nel momento attuale. Intanto perché vi è un'obiettiva difficoltà a individuare le norme da applicare. Poi perché, specie nelle materie contrattuali, assistiamo a un'ampia formazione di diritto spontaneo, di difficile conoscenza e dettato da gruppi di potere (e di pressione) molto forti e sovranazionali. Infine perché non si può essere certi del- 
la 'giustezza' delle leggi, peraltro spesso di difficile individuazione perché si devono applicare ad una negoziazione che travalica i confini degli Stati, rendendo arduo dire se utilizzare la legge dello Stato A o dello Stato B, di fronte alla globalizzazione dei processi di produzione e di commercializzazione delle merci, i quali interessano piú Stati.

Esemplare ed utile può apparire, per la soluzione delle liti mediante arbitrato e/ o conciliazione, l'esperienza romana della Repubblica, nella quale il processo civile era visto come regolamentazione privata degli assetti di interessi contrastanti: private erano le parti, privato era anche l'organo giudicante (arbiter o iudex). La Repubblica si preoccupava, attraverso un proprio magistrato (principalmente il praetor) di garantire il corretto svolgimento della procedura e l'imparzialità del giudicante.

In Italia nel 2009 si è inteso dare attuazione alla direttiva dell'UE 2008/52/CE dando delega al Governo affinché provvedesse ad introdurre e disciplinare la mediazione e conciliazione; assunto attuato con il successivo decreto legislativo del 4 marzo 2010, n. 28. La soluzione italiana è particolare, perché dichiaratamente rivolta ad accorciare i lunghi tempi dei processi, piú che alla ricerca della vera giustizia.

Manca all'appello la mediazione nel settore pubblico. Forse perché demandata alle varie figure di ombudsman che alcuni Paesi dell'UE conoscono da tempo ed è previsto all'interno della stessa UE con il nome di mediatore europeo. Su di essi occorre invece soffermarsi per avere il quadro completo della mediazione nell'ámbito dell'UE e per perseguire prospettive unificanti, conformi alla finalità di assicurare vera giustizia. 


\section{MEDIACJA I PROCES: KRYZYS PROCESU I UCIECZKA DO POJEDNANIA}

Mediacja w UE - W obecnych czasach uczestniczymy w ponownym odkryciu i odrodzeniu mediacji, na wszystkich poziomach - zarówno w stosunku ,publicznym” jak i ,prywatnym”. W szczególności zmierza się ku rozwiązaniu problemu dostępu do sprawiedliwości poprzez wprowadzenie mediacji i pojednania, jako form równoległych i pozasądowych przy rozstrzyganiu sporów. Jest to rozwiązanie opracowane w Unii Europejskiej.

Unia, po długiej i trudnej drodze, ustanowiła dyrektywę, która wskazuje odpowiedni kierunek i zobowiązuje państwa członkowskie do wprowadzania formy dobrowolnego i prewencyjnego pojednawczego rozstrzygania sporów. Chodzi tu o dyrektywę 2008/52/CE Parlamentu Europejskiego i Rady Europejskiej z dnia 21 maja 2008, która weszła w życie 12.6.2008. Zmierza ona do zwiększenia popularności mediacji jako metody rozwiązywania sporów w dziedzinie prawa cywilnego i handlowego oraz zobowiązuje państwa członkowskie do zrealizowania jej treści do maja 2011.

Dyrektywa stanowi przykład uznania prawa do sprawiedliwości jako prawa fundamentalnego i oznacza krok naprzód wobec Karty praw podstawowych UE z 2000 r., w której prawo do sprawiedliwości, przewidziane w rozdziale VI, było uznawane w związku z tradycyjną organizacją sądownictwa, czyli należącą do kompetencji sądów, z naciskiem położonym na procedury karne. Nowa dyrektywa bazuje na przeświadczeniu, że trudności w dostępie do sprawiedliwości w sprawach cywilnych i handlowych szkodzą fundamentalnym prawom i mogą wywołać skutek tak samo niszczący jak wyrok skazujący.

Unia Europejska, poprzez tę dyrektywę, nakłada na siebie obowiązek zapewnienia sprawiedliwego sądu stronom, które mogą przywiązywać do tego wagę i mają możliwości zorganizowania odrębnego procesu.

Sprawiedliwość osiągnięta w drodze mediacji wydaje się być podyktowana różnymi rodzajami świadomości, w zależności od zmian w systemach prawnych w obecnych czasach. Po pierwsze z przyczyny istnienia obiektywnych trudności w określeniu prawa właściwego do zastosowania. Po drugie, ponieważ, szczególnie w sprawach związanych z umowami, mamy do czynienia z szerokim pojęciem prawa zwyczajowego, w którym trudno się zorientować i które jest tworzone przez grupy trzymające władze (grupy nacisku) - bardzo silne i ponadnarodowe. W końcu, ponieważ nie można być pewnym ,sprawiedliwości” prawa, zresztą bardzo trud- 
nego do określenia, ponieważ trzeba je stosować do negocjacji, które przekraczają granice państw i bardzo trudno jest powiedzieć, czy należy stosować prawo państwa A czy państwa B - wobec globalizacji procesów produkcji i handlu towarami, które interesują większą ilość państw.

Doświadczenie Republiki Rzymskiej, w której proces cywilny był widziany jako prywatne uregulowanie sprzecznych interesów może służyć za wzór oraz być przydatne, dla rozwiązania sporów poprzez arbitraż i/lub pojednanie: prywatne były strony, prywatnym był również organ sądowniczy (arbiter lub iudex). Republice zależało aby, poprzez własnego sędziego (głównie praetora) zapewnić sprawny przebiegu postępowania i niezawisłość sędziego.

We Włoszech w 2009 r. postanowiono wprowadzić w życie dyrektywę UE 2008/52/CE upoważniając Rząd do ustalenia i przyjęcia zasad mediacji i pojednania; założenie zostało zrealizowane poprzez ustawę z dnia 4 marca 2010 r., nr 28. Włoskie rozwiązanie jest szczególne, ponieważ bardziej zmierza do skrócenia długiego czasu procesów, niż do poszukiwania prawdziwej sprawiedliwości.

Mediacji brakuje w sektorze publicznym. Być może dlatego, że to zadanie oddaje się różnym ombudsmanom urzędnikom, których niektóre państwa UE znają od dawna i który jest przewidziany wewnątrz samej Unii pod nazwą Europejskiego Rzecznika Praw Obywatelskich. Na tym należy się zatrzymać, aby uzyskać pełen obraz mediacji w Unii Europejskiej i aby dążyć do jednoczących perspektyw, w celu zapewnienia prawdziwej sprawiedliwości. 
Mediation in the EU. - Nowadays one takes part in the re-discovery and renaissance of mediation at all levels - both "public" and "private". In particular, it is intended to solve the problem of access to justice through the introduction of mediation and reconciliation, as parallel and out-of-court forms applied during disputes. This is the solution developed in the European Union.

The European Union, after a long and difficult road, laid down the directive, which shows the right direction in which to proceed and obliges member states to introduce forms of voluntary and preventive reconciliation to settle disputes.

With regard to Directive 2008/52/CE of the European Parliament and of the European Council of 21 May 2008, which entered into force 12.6.2008, the Directive aims to increase the popularity of mediation as a method of dispute resolution in both civil and commercial law and obliges member states to implement its contents by May 2011 .

The Directive, is an example of the recognition of the right to justice as being a fundamental right and is also a step forward in comparison to the regulation of the 2000 Charter of Fundamental Rights of the EU, in which the right to justice, as provided for in chapter VI, is recognized only in connection with the traditional organization of the judiciary, i.e. belonging to the competence of the courts, with the emphasis on criminal procedures. The new directive is based on the awareness that difficulties with access to justice in civil and commercial matters harm fundamental rights and that their effect could be equally as devastating as a conviction.

Key words: mediation, justice, civil process, ombudsman, European Union 